\title{
A PRINCIPAL COMPONENT ANALYSIS OF PROJECT PORTFOLIO MANAGEMENT PRACTICES
}

\author{
Matilda Alexandrova ${ }^{1, a, *}$ \\ ${ }^{1}$ University of National and World Economy, Studentski Grad, 1700 Sofia, Bulgaria \\ ${ }^{a}$ matildaa@unwe.bg \\ "Corresponding author
}

Cite as: Alexandrova, M. (2018). A principal component analysis of project portfolio management practices. Ekonomicko-manazerske spektrum, 12(2), 96-105.

Available at: dx.doi.org/10.26552/ems.2018.2.96-105

\begin{abstract}
In a turbulent business environment organizations are exposed to new opportunities but also various threats, e.g. financial, market, ecological, technological, politicalal, etc. In such highly competitive conditions the optimal utilization of resources emerges as a critical aspect of project management and its implementation in modern organizations. Nowadays project portfolio solutions are frequently indroduced in business practices. Deploying project portfolio management can facilitate the balance between effectiveness and efficiency. In the recent decades this approach has proliferated as a contemporary practice targeted to support the realization of the organizational strategy. The understanding of project portfolio management within the current study considers it as a centralized and balanced approach to the management of a set of projects constituting a portfolio, in order to achieve the strategic goals of the organization. This is accomplished by targeted processes - evaluation, selection, execution, prioritization, assessment, monitoring, and control of projects or programs - taking into account their alignment to the organizational strategy and contribution to the achievement of their objectives. The goal of the paper is to explore the major factors that favourably affect project portfolio management practices in Bulgarian project-oriented organizations using survey data from 184 respondents. Using an original research instrument, data about the implementation of project portfolio management were collected through purposive sampling of organizations that apply such an approach in their operations. Selected empirical results from a questionnaire survey of the factors of effective project portfolio management are presented regarding the practices in the studied organizations. A set of specific items concerning the project portfolio management practice are grouped into five major factors using principal component analysis. Each of these latent factors reflects a particular cluster of internal capabilities of the organizations to successfully implement project portfolio management in dynamic and complex business environment.
\end{abstract}

Keywords: project portfolio management, project-oriented organizations, principal component analysis, Bulgaria.

JEL Classification: M10, O22

\section{Introduction}

In the contemporary turbulent business environment, a new type of organizations systematically expands their activities, namely, the project-oriented organizations. They have 
proven to be more vital and effective in dealing with all challenges, opportunities, and threats emerging nowadays. Project-oriented organizations operating in the region of Central and Eastern Europe are especially concerned with the issues originating from their work in an international multi-project environment. In order to survive and develop in this sector any such organization must continuously introduce new managerial approaches, structures, and processes in order to maintain its competitiveness (Alexandrova et al., 2015).

In the modern conditions of economic, social, and cultural integration global networks have been developed concerning the communication, logistics, investment, and outsourcing operations. Moreover, an expansion of global project networks is observed where the so called global project is understood as a "temporary collaboration between organizations across nations and cultures with the intention to jointly deliver a unique product or service in a complex external context requiring relationship management" (Aarseth et al., 2013). This ultimately concerns individual professionals, project teams, and project-oriented companies in a variety of contexts related to the acquisition and implementation of specific knowledge, culture, skills, competences, and contemporary organizational practices in project management. Such an organizational practice is the project portfolio management which has emerged and proliferated in the recent decades. In many economic sectors, especially services, even a new phenomenon is noticed - named "projectification". It can be characterised by a "growing number of specialists organising their work in projects rather than on on-going functional basis" (Zhang et al., 2015).

The goal of the current study is to contribute to project portfolio management literature as a developing area by providing new knowledge about the major factors that favourably affect project portfolio management (PPM) practices on the basis of empirical data from projectoriented organizations in a CEE country. The literature review hereafter is based on selected titles from the specialized literature on PPM implementation, the factors of its effectiveness along with the challenges of the complex and turbulent business environment and internationalization of the project-based economy. These issues are of considerable importance regarding the operation of project-oriented organizations in international project networks which requires application of modern approaches such as PPM (Alexandrova, 2017).

PPM relates to the clustering of projects on the basis of their alignment to the organizational strategy. It is grounded on a centralized managerial policy for project selection, assessment, prioritization, and simultaneous execution. Strategic PPM is considered as one of the seven principles of the Strategic Project Leadership (Shenhar, 2004). Some authors link PPM with a complex of activities by which resources demands are affiliated to the availability of resources in the pursuit of the strategic objectives (Morris \& Jamieson, 2005). In this line, emerging necessities for organizational changes induce an expansion of the project-based activities which must be rapidly conducted, however, using limited resources (Alexandrova et al., 2017).

In the specialized literature on PPM two main streams are typically outlined. The first one emphasizes on normative frameworks and techniques oriented to evaluation, selection, and prioritization of projects in the portfolio (Martinsuo \& Lehtonen, 2007). The focus here is on the role of single projects, its characteristics and contribution to the portfolio. The second one shifts the attention more to portfolio structure, multiple-project factors, and the issues of managing the portfolio as a whole (Blomquist \& Muller, 2006). Killen et al. (2008) show that PPM and organizational learning can be determined as "dynamic capabilities" that boost the organization's potential to attain and preserve competitive advantages in a vigorous 
environment. Unlike single projects - which success can be linked to criteria like budget, time schedule, and quality of deliverables- the project portfolio assumes management of a set of interrelated projects affiliated to organizational strategy (Rank et al., 2015). The goals of PPM comprise maximization of the portfolio's value, aligning the portfolio with organizational strategy, and balancing the portfolio in order to achieve synergy (Cooper et al., 1999).

According to the specialized literature, several aspects of PPM can be outlined as internal capability factors facilitating the effectiveness of PPM, e.g. organizational knowledge (Martinsuo, 2013), targeted risk management (Teller, 2013), integrated information system for PPM (Jeffery \& Leliveld, 2003), structural alignment with strategy (Kaiser et al., 2015), project portfolio management office (Unger et al., 2012), HR competences and skills (Huemann, 2010), investments in human capital and employee training (Dobrovic et al., 2018), PPM international standards (Saynisch, 2010), etc.

For the goals of the current study -along with those PPM aspects identified in the scholarly literature- additional potential factors (internal capabilities) have been obtained as a result from a pilot study conducted by the author. In reality, a complex of diverse factors positively relates to PPM effectiveness through appropriate organizational structuring, portfolio balancing, and quality assurance. This way portfolio management can be aligned with the organizational strategy reflecting the dynamic nature in which projects are selected, prioritized, executed or discarded.

\section{Methodology}

According to the main goal of this paper, the empirical analysis attempts to identify the general factors of "internal capacity" of Bulgarian project-oriented organizations operating in a global environment and implementing a methodology for PPM. In practice, most of them realise international projects and/or projects commissioned by foreign contractors which provides opportunities and access to global resources and know-how.

The information basis for the analysis hereafter is data collected by a sample survey of 184 project-oriented organizations operating in Bulgaria in year 2017 conducted in the period 2016-2017. As far as there is no register or other statistical frame to facilitate a random drawing, the respondents have been selected by a purposive sampling scheme. A specifically designed questionnare has been developed and used for the goals of the survey. It was send to 200 respondents - project team members, project management experts, project managers, project portfolio managers, and representatives of the top management boards. The method of individual self-interview has been applied by participation in online survey or by submitting a filled questionnaire by email. Appropriate respondents have been reached using professional networks - LinkedIn, and Bulgarian Association for Project Management. All respondents have professional duties and competences in the area of project management performed in a multi-project global environment. Moreover, some of them have a key role in the management of a project portfolio operated by the respective organizations. Due to substantial non-response 16 questionnaires are excluded from data processing and analysis. The primary data collected by the survey instrument has been analysed by descriptive statistical methods, correlation analysis, and principal components analysis.

In order to evaluate the importance of the factors that favourably affect project portfolio management practices, a 15 questions are asked in the following form: „According to your experience, please evaluate each potential factor regarding the degree of its importance for 
PPM effectiveness in your organization when operating in a global environment". These potential factors have been derived as a result from a pilot study conducted in 2016 - mainly, indicators reflecting the ,internal capacity“ of a project-oriented organization for implementing a PPM approach in a global environment. The interview method that records the individual opinions about the issue of interest seems to be highly appropriate having in mind the research goals set for the study. Each question is of a close-end type and utilizes a 5rank scale about the degree of importance ranging from 1 (very low) to 5 (very high) with a midpoint of 3 (moderate). These evaluation ranks reflect the professional experience of the respondents from their work in a multi-project global environment.

Regarding the research goal of this study, the set of factors of PPM effectiveness are allocated into major clusters by application of a principal component analysis. As a traditional technique for multivariate analysis, it achieves a reduction of data dimensionality by extracting a small number of ,principal component“" variables while preserving, in largest possible extent, the degree of variation of the primary measured variables. On the other hand, the principal components should be less correlated between each other. Besides, they are extracted in a way that the first few components should represent a large share of the total variation observed in all primary variables. The reliability of implementation of this method is assessed by the Kaiser-Meyer-Olkin measure of sampling adequacy and Bartlett's test of sphericity (Hoffmann, 2016).

\section{Results}

\subsection{Profile of respondents}

The sample consists of respondents with different seniority and levels of professional and work experience in project-oriented organizations executing international projects. For each respondent a range of individual characteristics have been recorded (demographic and professional). A substantial share of them (over one third) indicated a long period of general work experience (over twenty years) whereas about $11 \%$ declared just a recent experience (up to five years). The professional experience in project management has been identified by the number of years working in project management (project team member, project office expert, project manager, project portfolio manager). The major share (about 60\%) is held by respondents with specific experience 6-15 years (Fig. 1). The correlation measured between the length of general and specific experience is quite high $(+0.84)$ which shows that relatively high share of the general work experience of respondents is actualy specialized in project management area.

One third of the espondents' act as project managers in their organization - a position which holds the largest share in the sample (Fig.2). About a quarter of the interviewed have project expert position followed by members of project teams $(21 \%)$, project office experts $(9 \%)$, and project portfolio managers $(8 \%)$. Albeit rarely, representatives of top management of project-oriented organisations $(3 \%)$ have also participated in the survey. 
Figure 1: Distribution of respondents by their general experience and professional experience in project management (\%)

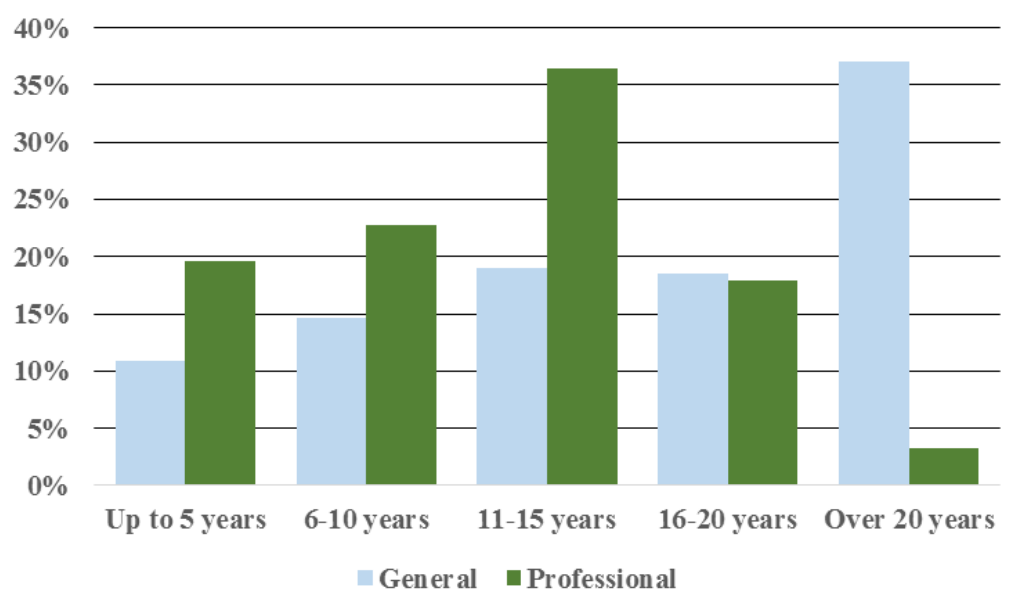

Source: Author's calculations

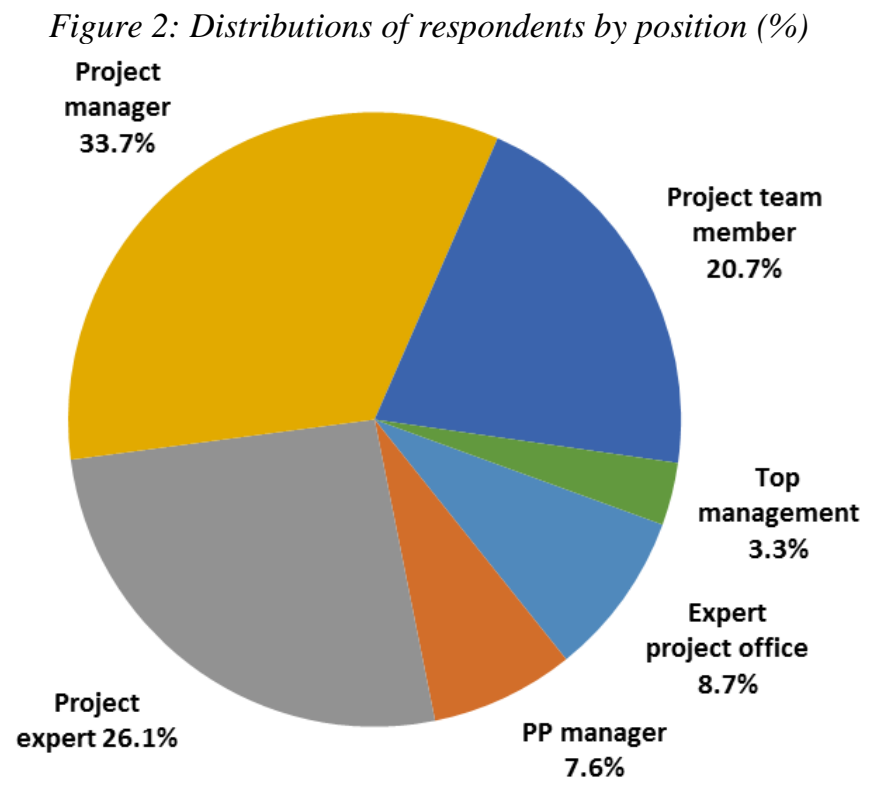

Source: Author's calculations

\subsection{Factor analysis}

In order to justify the utilization of principal component analysis as a tool for extracting latent factors, two methods are applied. According to the result obtained for Kaiser-MeyerOlkin measure of sampling adequacy $(\mathrm{KMO}=0.592)$ its value is greater than 0.5 so the sample size is adequate to implement the chosen method. The null hypothesis of the Bartlett's test of sphericity assumes that the correlation matrix of the 15 primary variables is an identity matrix. However, in order to use a factor analysis there must be significant correlations between two or more variables in the list; if this matrix is a unity one, then all correlations between any two variables would be zero. In our case, the level of significance of the Bartlett's test is less than 0.001 so the null hypothesis can be strongly rejected, i.e. the correlation matrix is significantly different from the identity matrix. In light of these results we assume that the application of principal component analysis is justified. 
Table 1: Communalities

\begin{tabular}{lcc}
\hline $\begin{array}{l}\text { Factors of the ,internal capacity“ of the organization for implementing a PPM } \\
\text { approach }\end{array}$ & Initial & Extraction \\
\hline Project-oriented organizational culture (V1) & 1.000 & 0.783 \\
Clearly defined rules/processes for PPM (V2) & 1.000 & 0.679 \\
Clearly defined roles and responsibilities in PPM (V3) & 1.000 & 0.825 \\
Integrated information system for PPM (V4) & 1.000 & 0.687 \\
Organizational knowledge about business / market (V5) & 1.000 & 0.674 \\
Organizational knowledge about PPM International Standards (V6) & 1.000 & 0.762 \\
Education / professional qualification of HR (V7) & 1.000 & 0.383 \\
Specific PPM professional qualification of HR (V8) & 1.000 & 0.709 \\
Specific PPM experience of HR (V9) & 1.000 & 0.665 \\
Implementation of targeted risk management (V10) & 1.000 & 0.639 \\
Fully functional Project Management Office (V11) & 1.000 & 0.779 \\
Effective communications between stakeholders (V12) & 1.000 & 0.739 \\
Setting priorities for projects selection (V13) & 1.000 & 0.701 \\
Organizational structure reflecting PPM processes (V14) & 1.000 & 0.713 \\
Subordination of PPM processes to the organizational strategy (V15) & 1.000 & 0.729
\end{tabular}

Source: Author's calculations

Table 2: Principle components extracted

\begin{tabular}{lccccc}
\hline $\begin{array}{l}\text { Factors of the ,internal capacity“ of the organization for } \\
\text { implementing a PPM approach }\end{array}$ & PC1 & PC2 & PC3 & PC4 & PC5 \\
\hline Project-oriented organizational culture (V1) & $\mathbf{0 . 8 7 2}$ & 0.111 & 0.044 & 0.088 & -0.022 \\
Organizational knowledge about PPM International Standards & $\mathbf{0 . 7 6 9}$ & 0.175 & 0.160 & 0.144 & 0.305 \\
(V6) & $\mathbf{0 . 7 0 4}$ & 0.140 & -0.073 & 0.371 & -0.129 \\
Organizational knowledge about business / market (V5) & $\mathbf{0 . 5 8 6}$ & 0.483 & 0.149 & 0.142 & 0.138 \\
Implementation of targeted risk management (V10) & & & & & \\
Subordination of PPM processes to the organizational & 0.085 & $\mathbf{0 . 8 2 1}$ & 0.203 & 0.050 & 0.062 \\
strategy (V15) & 0.447 & $\mathbf{0 . 6 8 7}$ & -0.161 & -0.025 & -0.118 \\
Organizational structure reflecting PPM processes (V14) & 0.461 & $\mathbf{0 . 5 6 4}$ & 0.260 & 0.305 & 0.297 \\
Fully functional Project Management Office (V11) & -0.199 & 0.166 & $\mathbf{0 . 7 8 2}$ & 0.167 & -0.183 \\
Effective communications between stakeholders (V12) & 0.287 & -0.305 & $\mathbf{0 . 7 5 1}$ & -0.263 & 0.128 \\
Clearly defined roles and responsibilities in PPM (V3) & 0.185 & 0.340 & $\mathbf{0 . 7 0 8}$ & 0.165 & 0.012 \\
Clearly defined rules/processes for PPM (V2) & 0.096 & 0.114 & 0.073 & $\mathbf{0 . 8 2 2}$ & 0.069 \\
Specific PPM professional qualification of HR (V8) & 0.317 & -0.015 & 0.022 & $\mathbf{0 . 7 6 8}$ & 0.102 \\
Setting priorities for projects selection (V13) & 0.158 & -0.168 & -0.065 & -0.017 & $\mathbf{0 . 7 8 0}$ \\
Specific PPM experience of HR (V9) & -0.133 & 0.236 & -0.085 & 0.019 & $\mathbf{0 . 7 7 9}$ \\
Integrated information system for PPM (V4) & 0.096 & 0.042 & 0.103 & 0.334 & $\mathbf{0 . 5 0 0}$ \\
Education / professional qualification of HR (V7) & &
\end{tabular}

Source: Author's calculations. Conditions: eigenvalue of 1.0; Varimax rotation with Kaiser Normalization

As a first step of the principal component analysis the communalities of the primary variables are estimated. Each communality explains the amount of the variance of the respective primary variable that can be explained by the extracted five latent factors. It should be noted that after the extraction a residual share of the total variance has stayed unexplained (in our case, about 30\%) and thus some latent factors are lost. Most of the communalities 
have values between 0.67 and 0.80 which shows a good intrinsic interrelations between the primary variables and the underlying factors.

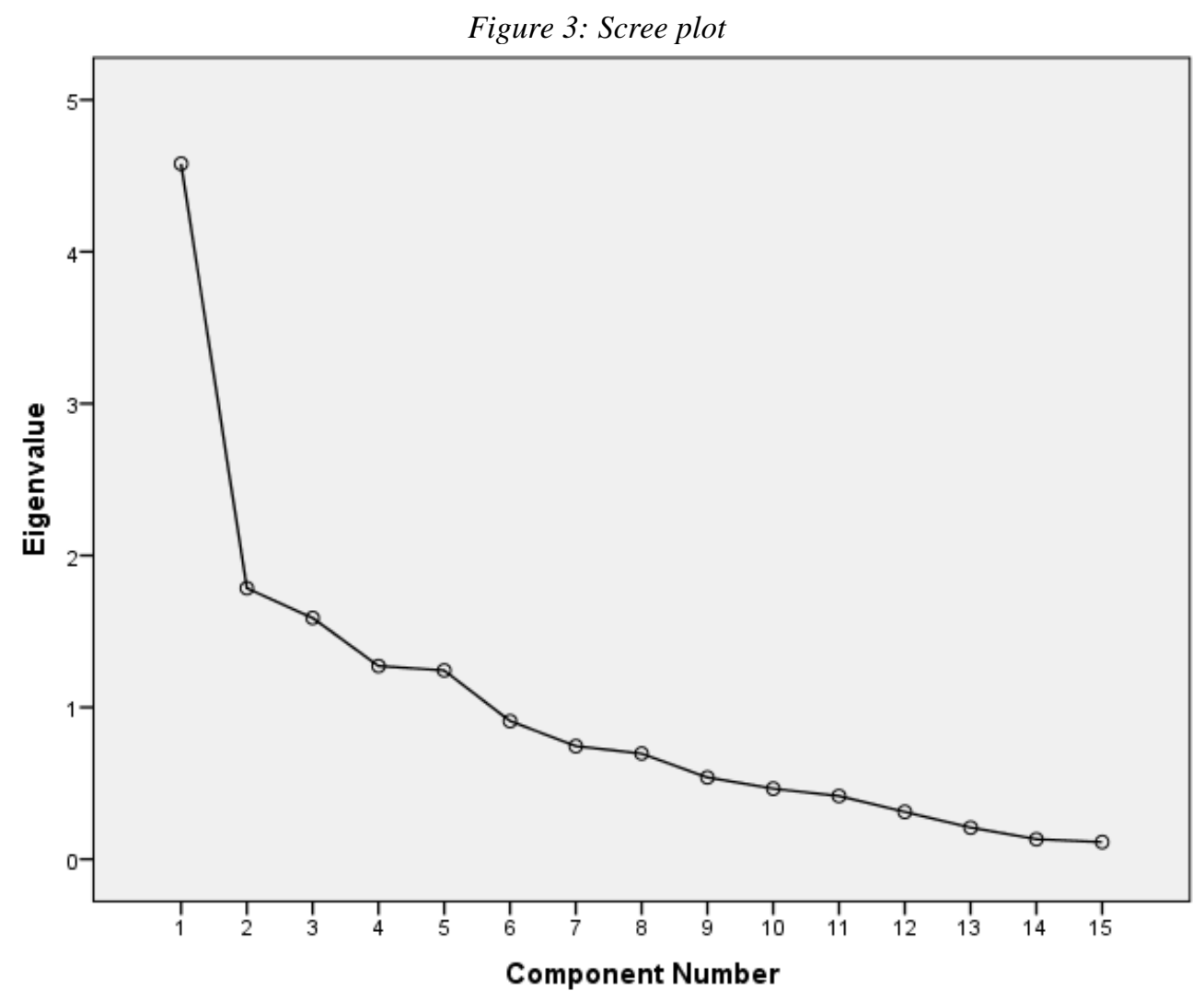

Source: Author's calculations

Table 2 presents the rotated component matrix obtained by applying the latent root criterion for extracting principal components. As shown on the scree plot (Fig.3) five components having eigenvalues over 1.0 were extracted using a factor loading of 0.50 as cutoff point. These five components have explained $69.8 \%$ of the total variation of the primary variables V1-V15 which can be assumed as sufficient, with a balanced distribution of the variance explained (PC1 accounted for 19.5\%, PC2- 14\%, PC3- 12.6\%, PC4- 11.9\%, PC5-11.8\%). The composition of each component is determined by applying the traditional threshold of 0.5 for the factor loading of any variable. The interpretation of the results should generally be cautious since a combination of primary variables in a component (obtained just due to high loadings) sometimes might be challenging to decipher.

\section{Discussion}

After a critical assessment of the nature of the primary variables within each component, a plausible interpretation is derived in respect of the latent factors of interest. The names reflect the consistency of the interrelation between the respective characteristics of project portfolio management aspects:

- PC1 could be labeled as "Organizational Culture and Knowledge";

- PC2 could be labeled as "Organizational Strategy, Structure, and Processes";

- PC3 could be labeled as "Communications, Formalization, and Standardization";

- PC4 could be labeled as "Prioritization and Professional Competences"; 
- PC5 could be labeled as "HR and IT Infrastructures".

Principal component 1 is themed as "Organizational Culture and Knowledge" due to the incorporation of four variables: Project-oriented organizational culture (V1), Organizational knowledge about PPM International Standards (V6), Organizational knowledge about business / market (V5), and Implementation of targeted risk management (V10). This result shows that the importance of cultural and knowledge factors of successful PPM implementation plays a leading role for the project-based organization. This result corresponds to the statement that a great challenge here is to stimulate a positive climate and supportive culture for the implementation of PPM methods and knowledge sharing between projects in the portfolio (Cooper et al., 2001). Moreover, in conditions of growing complexity and dynamics, targeted risk mitigation is crucial for the survival and development of the project-oriented organizations.

Principal component 2 is named as "Organizational Strategy, Structure, and Processes" as far as the integrated variables are: Subordination of PPM processes to the organizational strategy (V15), Organizational structure reflecting PPM processes (V14), and Fully functional Project Management Office (V11). It is notable that the variables with high loadings to this factor relate to the degree of alignment of the structure of the project oriented organization to its strategy. Suitable organizational structure should enable execution of the projects subjected to organizational strategy (Lanka, 2007). In this respect, the appropriately design of the organizational structure facilitates the effective execution of the PPM processes. This is especially valid for the modern organizational forms like the establishment of a Project Management Office.

Principal component 3 is called "Communications, Formalization, and Standardization" because of the high loadings of the following variables: Effective communications between stakeholders (V12), Clearly defined roles and responsibilities in PPM (V3), and Clearly defined rules/processes for PPM (V2). Here several formal aspects of PPM implementation, namely communications aligned with the structure and hierarchy of PPM rules and procedures. The established by formal rules interrelations and communications between stakeholders provide opportunities for standardization of PPM processes, economies of time, costs, and other resources. Our study results confirm previous research which shows that the formalization of the management practices for both individual projects and the overall portfolio indisputably relates to project portfolio success (Teller et al., 2012).

Principal component 4 can be entitled "Prioritization and Professional Competences" due to the involvement of the following two variables: Specific PPM professional qualification of HR (V8) and Setting priorities for projects selection (V13). This component mixes a human factor associated to PPM professional competences with one of the most important functions of PPM, namely, project prioritization. These two aspects are considered as crucial for the successful implementation of PPM in a project-oriented organization having in mind that proper selection of priority projects is hardly possible without high professional qualifications of the PPM personnel. Allocating the right human resources, with their professional competencies, to the different project teams is considered as critical from the point of view of associating the short-term planning with strategical alignment, assuming the priorities set to the project portfolio (Hendriks et al., 1999).

Principal component 5 deals with aspects under the heading "HR and IT Infrastructures" since the incorporated variables are: Specific PPM experience of HR (V9), Integrated information system for PPM (V4), and Education / professional qualification of HR (V7). People are the predominant resource in a project-oriented organization, and there is a positive 
association between favorable HR management and successful PPM implementation. Moreover, in conditions of high competitiveness effective utilization of HR potential is impossible without the usage of an integrated information system for PPM. This definitely relates to improving the performance of the human capital that needs to operate in real time, forming virtual teams, and rapidly communicating when executing projects in the digital era. Many authors outline that specialised software for PPM is crucial for diminishing the duration of information processing, distribution, and provision to support the project portfolio decision making (De Reyck et al., 2005).

\section{Conclusion}

In light of contemporary globalization processes a range of factors determine the effective implementation of PPM practices in modern project-oriented organizations. These factors are expected to play a core role in the further development of PPM worldwide. Still, there is a shortage of comprehensive knowledge as well as unexplored challenges concerning the execution of international projects in the global environment by such organizations. Thus, the current study provides new insights and understandings of the impact of a set of factors reflecting the internal capabilities of project-oriented organizations. The identified fifteen variables have been clustered into five common factors facilitating the success of PPM practices. So, a key contribution of the paper is revealed by the application of principal component analysis accompanied by carefully derived conception about the nature of the extracted factors and their role for the PPM implementation in a global competitive environment.

The study also provides certain implications about the current stage of project portfolio management research in Bulgaria. The emerging practices of introduction and enhancement of PPM in Bulgarian project-oriented organizations require further investigation of various aspects of its performance, effectiveness, and overall success. The presented survey results provide ground to the statement that the spread of PPM practices in the country is related to the acquisition of organizational knowledge, implementation of international standards, effective communications, and utilization of human resources with professional competences.

\section{References}

Aarseth, W., Rolstadås, A. \& Andersen, B. (2013). Managing Organizational Challenges in Global Projects. International Journal of Managing Projects in Business, 7(1), 103-132.

Alexandrova, M. (2017). Project Portfolio Management Processes: Survey Evidence from Bulgarian ProjectOriented Organizations. Yearbook of UNWE, 1, 73-85.

Alexandrova, M., Kuzmanova, M. \& Atanassov, A. (2017). Change Management Through Projects: Issues and Perspectives. Proceedings of ETAP 2017 Conference "Economic Theory and Practice”, Banska Bystrica, Slovakia, 12-21.

Alexandrova, M., Stankova, L. \& Gelemenov, A. (2015). The Role of Project Office for Project Portfolio Management. Economic Alternatives, 1, pp.19-30.

Blomquist, T. \& Muller, R. (2006). Practices, Roles, and Responsibilities of Middle Managers in Program and Portfolio Management. Project Management Journal, 37(1), 52-66.

Cooper, RG., Edgett, S.J. \& Kleinschmidt, E.J. (1999). New Product Portfolio Management: Practices and Performance. Journal of Production and Innovation Management, 16(4), 333-350.

Cooper, R., Edgett, S. \& Kleinschmidt, E. (2001). Portfolio Management for New Products: Results of an Industry Practices Study. R\&D Management, 31(4), 361-380.

De Reyck, B. et al. (2005). The Impact of Project Portfolio Management on Information Technology Projects. International Journal of Project Management, 23(7), 524-537. 
Dobrovic, J., Lambovska, M., Gallo, P. \& Timkova, V. (2018). Non-Financial Indicators and Their Importance in Small and Medium-Sized Enterprises. Journal of Competitiveness, 10(2), 41-55.

Hendriks, M., Voeten, B. \& Kroep, L. (1999). Human Resource Allocation in a Multi-Project R\&D Environment. Resource Capacity Allocation and Project Portfolio Planning in Practice. International Journal of Project Management, 17(3), 181-188.

Hoffmann, J.P. (2016). Principal Components and Factor Analysis. Regression Models for Categorical, Count, and Related Variables: An Applied Approach. Oakland, CA: University of California Press, 243-268.

Huemann, M. (2010). Considering Human Resource Management when developing a project-oriented company: Case study of a telecommunication company. International Journal of Project Management, 28(4), 361-369.

Jeffery, M. \& Leliveld, I. (2004). Best Practices in IT Portfolio Management. MIT Sloan Management Review, 45(3), 41-49.

Kaiser, M.G., El Arbi, F. \& Ahlemann, F. (2015). Successful Project Portfolio Management Beyond Project Selection Techniques: Understanding the Role of Structural Alignment. International Journal of Project Management, 33(1), 126-139.

Killen, C.P., Hunt, R.A. \& Kleinschmidt, E.J. (2008). Learning Investments and Organizational Capabilities: Case Studies on the Development of Project Portfolio Management Capabilities. International Journal of Managing Projects in Business, 1(3), 334-351.

Lanka, M.C. (2007). Strategically Aligning your Project Portfolios: Introducing a New Paradigm in Project Portfolio Management. Paper presented at PMI Global Congress 2007 North America, Atlanta, GA. Newtown Square, PA: Project Management Institute.

Martinsuo, M. (2013). Project Portfolio Management in Practice and in Context. International Journal of Project Management, 31(6), 794-803.

Martinsuo, M. \& Lehtonen, P. (2007). Role of Single-Project Management in Achieving Portfolio Management Efficiency. International Journal of Project Management, 25(1), 56-65.

Morris, P. \& Jamieson, A. (2005). Moving from Corporate Strategy to Project Strategy. Project Management Journal, 36(4), 5-18.

Rank, J., Unger, B.N. \& Gemünden, H.G. (2015). Preparedness for the Future in Project Portfolio Management: The Roles of Proactiveness, Riskiness and Willingness to Cannibalize. International Journal of Project Management, 33(8), 1730-1743.

Saynisch, M. (2010). Mastering Complexity and Changes in Projects, Economy, and Society Via Project Management Second Order (PM-2). Project Management Journal, 41(5), 4-20.

Shenhar, A. (2004). Strategic Project Leadership ${ }^{\circledR}$ : Toward a Strategic Approach to Project Management. $R \& D$ Management, 34(5), 569-578.

Teller, J. (2013). Portfolio Risk Management and its Contribution to Project Portfolio Success: An Investigation of Organization, Process, and Culture. Project Management Journal, 44(2), 36-51.

Teller, J., Unger, B.N., Kock, A. \& Gemünden, H.G. (2012). Formalization of Project Portfolio Management: The Moderating Role of Project Portfolio Complexity. International Journal of Project Management, 30(5), 596-607.

Unger, B.N., Gemunden, H.G. \& Aubry, M. (2012). The Three Roles of a Project Portfolio Management Office: Their Impact on Portfolio Management Execution and Success. International Journal of Project Management, 30(5), 608-620.

Zhang, Y., Marquis, C., Filippov, S., Haasnoot, H.-J. \& Van der Steen, M. (2015). The Challenges and Enhancing Opportunities of Global Project Management: Evidence from Chinese and Dutch Cross-Cultural Project Management. Harvard Business School Working Paper (15-063). 